Journal: Journal of Pharmaceutical and Biomedical Analysis

\title{
Quantitative Determinations Using Portable Raman Spectroscopy
} Chelliah V. Navin, Chaitanya Tondepu, Roxana Toth, Latevi S. Lawson and Jason D.
Rodriguez*

Division of Pharmaceutical Analysis, Center for Drug Evaluation and Research, US Food and Drug Administration, 645 S. Newstead Ave Saint Louis, MO 63110

*EMAIL: Jason.Rodriguez@fda.hhs.gov

CORRESPONDING AUTHOR INFORMATION: Tel (314) 539-3855 Fax (314) 539-2113 


\begin{abstract}
A portable Raman spectrometer was used to develop chemometric models to determine percent (\%) drug release and potency for $500 \mathrm{mg}$ ciprofloxacin $\mathrm{HCl}$ tablets. Parallel dissolution and chromatographic experiments were conducted alongside Raman experiments to assess and compare the performance and capabilities of portable Raman instruments in determining critical drug attributes. All batches tested passed the 30 minute dissolution specification and the Raman model for drug release was able to essentially reproduce the dissolution profiles obtained by ultraviolet spectroscopy at $276 \mathrm{~nm}$ for all five batches of the $500 \mathrm{mg}$ ciprofloxacin tablets. The five batches of $500 \mathrm{mg}$ ciprofloxacin tablets also passed the potency (assay) specification and the \% label claim for the entire set of tablets run were nearly identical, $99.4 \pm 5.1$ for the portable Raman method and $99.2 \pm 1.2$ for the chromatographic method. The results indicate that portable Raman spectrometers can be used to perform quantitative analysis of critical product attributes of finished drug products. The findings of this study indicate that portable Raman may have applications in the areas of process analytical technology and rapid pharmaceutical surveillance.
\end{abstract}




\section{Introduction}

The traditional paradigm of pharmaceutical surveillance is to collect samples in the field and send them to the laboratory for analyses[1] such as dissolution and chromatography $[2,3]$. This workflow has a longstandinghistory $[4,5]$ and demonstrated record of success. However, the existing process requires updating in order to reflect the needs of the $21^{\text {st }}$ century pharmaceutical analytical laboratory, such as enhancing the speed and efficiency of product-testing. One rapid and versatile technique that is becoming increasingly common in the pharmaceutical laboratory is portable Raman spectroscopy, which has gained popularity in field screening over the past decade due, in part, to being widely commercially-available[6]. Portable Raman spectrometers are used in qualitative experiments for identification, and are capable of analyzing samples in less than one minute [7-9]. Quantitative studies using Raman are traditionally performed in combination with chemometric-based approaches[10], and have been used for a wide range of applications such as polymorph quantification[11] and adulterant detection[12, 13].

To better understand the capability of portable Raman spectroscopy as a quantitative analytical tool[14], we report our efforts to study the drug release and potency of U.S. FDA-approved commercially-available 500 mg ciprofloxacin $\mathrm{HCl}$ tablets. Ciprofloxacin is an important antibiotic drug used for prophylaxis and treatment of anthrax, plague, and tularemia outbreaks[15]. During the 2001 anthrax crisis, ciprofloxacin received significant attention as a first-line of treatment for inhalation anthrax exposure and is one of the most commonly stockpiled drugs[16]. In this proof of concept effort, we performed parallel Raman analysis with traditional dissolution and HPLC testing on the same samples to make direct comparisons between the portable Raman spectroscopic results and traditional laboratory approaches.

\section{Materials and Methods}

$\underline{\text { Samples }}$

A total of 60 tablets were chosen for this study by randomly selecting 12 tablets from five different batches of commercially-available $500 \mathrm{mg}$ ciprofloxacin $\mathrm{HCl}$ tablets (NDC\# 55111-127-01). The original tablet pool of 60 was split into two groups of 30 used respectively for dissolution and HPLC assays.

Calibration Curves for Dissolution (Release) Testing

Six different calibration samples of ciprofloxacin $\mathrm{HCl}$ solutions, ranging from 0.1 to $0.85 \mathrm{mg} / \mathrm{ml}$, were prepared by dissolving ciprofloxacin $\mathrm{HCl}$ monohydrate USP reference standard in $0.01 \mathrm{~N} \mathrm{HCl}$. The solutions were used "as is" for Raman but were diluted 100x for ultraviolet (UV) analysis at $276 \mathrm{~nm}$. All samples were placed in Brand UV disposable cuvettes (Brand GMBH) and analyzed within 24 hours of preparation. The disposable cuvettes 
have two path length options: $0.5 \mathrm{~cm}$ and $1.0 \mathrm{~cm}$, of which we chose the $1.0 \mathrm{~cm}$ length for Raman analysis. Each sample was placed in its own cuvette and cuvettes were not reused. There were no discernable differences detected from using disposable cuvettes for any of the work reported here. The Raman spectral collection was performed in triplicate using a $785 \mathrm{~nm}$ excitation Enwave EZ Raman-1 portable spectrometer (TSI, Inc. formerly Enwave Optronics, Inc.) using the vial/cuvette sampling accessory with the cuvette in the $1.0 \mathrm{~cm}$ path length orientation. The $\mathrm{CCD}$ detector was operated at $-50^{\circ} \mathrm{C}$ and the acquisition time was set to 40 seconds for all samples used to assess release. Spectra were collected from $250-2000 \mathrm{~cm}^{-1}$. Then the spectra were analyzed using PLS Toolbox (Version 8.0.2, Eigenvector Research, Inc.) to obtain a calibration curve based on the partial least squares (PLS) linear regression algorithm. A summary of the parameters used for the linear calibration model is given in Table 1. The Raman spectral range used for method development was selected based on the region where ciprofloxacin $\mathrm{HCl}$ peaks are most prominent and trends based on concentration were observed. The number of latent variables (LVs) was chosen based on careful comparison of the root mean squared error of calibration (RMSEC) and root mean squared error of cross validation (RMSECV) trends.

The 100x diluted standard solutions were analyzed with an Agilent 8453 UV-Visible spectrometer (Agilent Technologies, Inc.) from 200-800 $\mathrm{nm}$ through the short path length of the cuvette $(0.5 \mathrm{~cm})$. The absorbance values for $276 \mathrm{~nm}$ were used to establish the linear calibration curve. The calibration curve for the UV measurements had a $\mathrm{R}^{2}$ value of 0.999 and slope of $0.545 \mathrm{ml} \cdot \mathrm{mg}^{-1} \cdot$ [absorbance units].

All values reported for $\%$ drug release are based on the amount of ciprofloxacin. Percent (\%) drug release for the profile measurements have been calculated without aliquot replacement according to established approaches[17].

\section{Dissolution (Release) Testing}

The first group of 30 tablets was analyzed for \% drug release based on the dissolution methods available in the United States Pharmacopeia (USP) monograph for ciprofloxacin tablets[18]. The dissolution testing was conducted with USP Dissolution Apparatus II (paddle method) on a Hanson Vision Elite 8 (Hanson Research Corp.) instrument. The paddles were set to $50 \mathrm{rpm}$ in $900 \mathrm{ml} 0.01 \mathrm{~N} \mathrm{HCl}$ solution at $37^{\circ} \mathrm{C}$. Sample aliquots $(10 \mathrm{ml})$ were manually withdrawn using a $15 \mathrm{ml}$ syringe from the dissolution vessel at 10, 20, 30 and 60 minutes to obtain a release profile for the batches. The time points were picked to facilitate manual collection, filtration, and 
preparation of the samples for spectroscopic analysis. The aliquots were filtered through a $0.45 \mu \mathrm{m}$ polyvinylidene fluoride (PVDF) media filter with polypropylene housing (25 mm GD/X Disposable filter device). Approximately $1.5 \mathrm{ml}$ of the filtered solution was transferred to a Brand UV disposable cuvette (Brand GMBH). A second set of samples was prepared from the filtered aliquots which was diluted 100x for analysis by UV spectroscopy at $276 \mathrm{~nm}$. The UV-Vis dilutions were prepared using $0.01 \mathrm{~N} \mathrm{HCl}$ (dissolution medium). Raman and UV analysis were performed as described above for the calibration samples.

\section{Raman Calibration for HPLC (Potency) Testing}

Nine different calibration samples of ciprofloxacin $\mathrm{HCl}$ solutions, ranging from 0.1 to $1.0 \mathrm{mg} / \mathrm{ml}$, were prepared by dissolving ciprofloxacin $\mathrm{HCl}$ monohydrate USP reference standard in a solution containing $0.025 \mathrm{M}$ ophosphoric acid and acetonitrile in an 87:13 ratio. The standard solutions were filtered through a $0.45 \mu \mathrm{m}$ filter and placed in Brand UV disposable cuvettes (Brand GMBH). All samples were analyzed within 72 hours of preparation and stored in the refrigerator when not in use. The Raman spectral collection was performed in triplicate using the Enwave EZ-Raman $\mathrm{H}$ portable spectrometer, as described above, using the same parameters with the exception of integration time, which was set to 30 seconds for all of the samples used to assess potency. The less integration time required between the potency and release experiments was due to the difference in the Raman scattering signal from the potency sample matrix which included acetonitrile, a strong Raman scatterer. The lower integration time was needed to avoid saturation of the detector. The $1.0 \mathrm{~cm}$ path length of the cuvette was used. The samples were analyzed using PLS Toolbox to obtain a calibration model based on the PLS linear regression algorithm, as described above. A summary of the parameters used for the linear calibration model is given in Table 1. All values reported for \% label claim (\% LC) are based on the amount of ciprofloxacin present and label claim (500 mg ciprofloxacin) listed on the bottle.

\section{HPLC Sample Preparation:}

The second group of 30 tablets was analyzed to determine the assay of the ciprofloxacin based on the HPLC methods available in the USP monograph for Ciprofloxacin[19]. A diluent was prepared in the ratio 13:87 of acetonitrile and $0.025 \mathrm{M}$ o-phosphoric acid adjusted to $\mathrm{pH} 2$ with triethylamine. Each of the 30 ciprofloxacin tablets were separately dissolved to a concentration of $0.2 \mathrm{mg} / \mathrm{ml}$ in the diluent and filtered using a $0.45 \mu \mathrm{m}$ PVDF filter. Acetonitrile was mixed with $0.025 \mathrm{M}$ o-phosphoric acid adjusted to $\mathrm{pH} 3$ with triethylamine, in ratio 13:87 to create the mobile phase. Prior to running the ciprofloxacin samples on HPLC, USP ciprofloxacin ethylenediamine and 
ciprofloxacin $\mathrm{HCl}$ standards were run through the HPLC column to confirm the system's suitability. The percentage assay of ciprofloxacin tablets was calculated based on Equation 1:

$$
\text { Result }=\left(\mathrm{r}_{\mathrm{u}} / \mathrm{r}_{\mathrm{s}}\right) \times\left(\mathrm{C}_{\mathrm{s}} / \mathrm{C}_{\mathrm{u}}\right) \times\left(\mathrm{M}_{\mathrm{r} 1} / \mathrm{M}_{\mathrm{r} 2}\right) \times 100
$$

$\mathrm{r}_{\mathrm{u}}$ : peak response from the sample solution

$\mathrm{r}_{\mathrm{s}}$ : peak response from the standard solution

$\mathrm{C}_{\mathrm{s}}$ : concentration of USP ciprofloxacin hydrochloride RS in the Standard solution (mg/ml), calculated on the anhydrous basis

$\mathrm{C}_{\mathrm{u}}$ : nominal concentration of ciprofloxacin in the sample solution $(\mathrm{mg} / \mathrm{ml})$

$\mathrm{M}_{\mathrm{r} 1}$ : molecular weight of ciprofloxacin, 331.34

$\mathrm{M}_{\mathrm{r} 2}$ : molecular weight of anhydrous ciprofloxacin hydrochloride, 367.81

\section{HPLC Components and Parameters}

The HPLC analyses were conducted with an Atlantis C18 $5 \mu \mathrm{m}$ column $(4.6 \mathrm{~mm}$ x $25 \mathrm{~cm})$. The HPLC experiments were performed with a run time of 20 minutes at $1.5 \mathrm{ml} / \mathrm{min}$ flow rate and an injection volume of $10 \mu 1$ at a column temperature of $30^{\circ} \mathrm{C}$ on an Agilent 1290 Infinity II LC system (Agilent Technologies, Inc., Santa Clara, CA). The samples were analyzed using a diode array detector with a UV absorbance of $278 \mathrm{~nm}$.

\section{Results and Discussion}

The Raman spectra for the dissolution standard solutions are shown in Figure 1 in the $1100-1800 \mathrm{~cm}^{-1}$ region. There are four regions where there is clear ciprofloxacin concentration dependence on the spectra at 1274 , 1343, 1391 and $1631 \mathrm{~cm}^{-1}$. The most notable is the peak at $1391 \mathrm{~cm}^{-1}$ where the spectral intensity trends closely follow the known concentration trends of the standards. The other three regions are somewhat less prominent but also show strong dependence on ciprofloxacin concentration, although it is not necessarily as clear that the ordering is followed as closely as the $1391 \mathrm{~cm}^{-1}$ peak. It should be noted that, while these regions clearly depict the spectral changes as a function of ciprofloxacin, method development encompassed the entire spectral region from 1100-1800 $\mathrm{cm}^{-1}$, as specified in Table 1. The PLS algorithm includes all spectral features even where there are not clear trends observed by the un-aided eye. The Raman spectra for the reference standard for solid-state ciprofloxacin $\mathrm{HCl}$ is shown for comparison. The aforementioned regions appear to correspond well with strong features in the solid-state reference standard Raman spectrum especially for the $\sim 1391 \mathrm{~cm}^{-1}$ and $1631 \mathrm{~cm}^{-1}$ peaks in the ciprofloxacin solutions which correspond to strong bands at 1386 and $1623 \mathrm{~cm}^{-1}$ in the reference standard.

The fit acquired for the Raman dissolution calibration model and associated figures of merit are listed in

Table 1 . The high degree of linearity between the measured concentration and the predicted concentration based on 
cross validation is indicated by the $\mathrm{R}^{2}$ value of 0.999 . Similar performance is observed at both extreme ends of the model and the range was selected such that the theoretical concentration for a $500 \mathrm{mg}$ ciprofloxacin tablet in the dissolution vessel $(0.56 \mathrm{mg} / \mathrm{ml})$ would fall roughly in the middle of the calibration sample concentration range. The range indicates that the model would likely work for other dosage strength tablets which are commonly available for ciprofloxacin in the 100-1000 $\mathrm{mg}$ range.

For each of the five batches run, the calibration model was used to predict the concentration for the dissolution aliquots at different time points to obtain a dissolution profile for each batch. The Raman dissolution profile was then compared to the dissolution profile obtained by the USP method using UV quantitation at 276 $\mathrm{nm}[18]$. The dissolution profiles for each of the five batches are shown in Figure 2. The data points are based on the average result for each of the six tablets used from each batch. The error bars represent the standard deviation for the results of the six tablets. The results are also listed in Table 2. All of the batches passed the specification for not less than (NLT) $80 \%$ of the labeled amount of ciprofloxacin at the 30 minute time point. All of the profiles were based on the same dissolution aliquots but differ in the method used for quantification of the \% ciprofloxacin drug release-either Raman or UV. Where release values are slightly above $100 \%$, the respective models predict more ciprofloxacin is present than the theoretical amount, which may occur due to normal batch to batch variability in the manufacturing process. The profiles show remarkable similarity within the standard deviation error bars for four of the five batches: Batch 1, Batch 3, Batch 4 and Batch 5. The lone exception is Batch 2, shown in Figure 2B where the profiles are qualitatively similar but somewhat offset outside the standard deviation uncertainties for each of the points after 10 minutes. It is also noteworthy that for all the batches, the Raman data produced tighter results for each time points as evidenced by the relatively small error bars compared to the UV data. This might be indicative of the sample preparation necessary for the UV samples compared to the Raman samples which require no further dilution. Collectively, the results show that portable Raman can be used to essentially obtain the same results as the traditional method-UV. The larger ramification for the dissolution data indicate the potential to use portable Raman instruments through immersion or fiber optic probes—-for example — to determine real time \% drug release without further sample preparation such as dilution of samples as is often needed for UV to overcome concerns about saturating UV detectors.

The Raman spectra for the HPLC standard solutions are shown in Figure 3 in the $1100-1800 \mathrm{~cm}^{-1}$ region. The standard solutions for HPLC were prepared in solutions containing o-phosphoric acid and acetonitrile, the latter, 
which is a strong Raman scatterer, and dominates the Raman spectra. Nevertheless, there are two regions just below $\sim 1390$ and at $\sim 1630 \mathrm{~cm}^{-1}$ where there are clear differences as a function of varying ciprofloxacin concentration. These regions are shown in further detail in Figure 3B, which gives a zoomed-in view of the Raman spectra. As previously noted for the dissolution experiments, method development encompassed the entire spectral region from $1100-1800 \mathrm{~cm}^{-1}$, as specified in Table 1. The solid state reference standard Raman spectrum is given in Figure 3A to facilitate comparison with the standard solutions. The two most intense peaks in the solid state spectrum at 1386 and $1623 \mathrm{~cm}^{-1}$ correspond closely to the regions where the ciprofloxacin concentration dependence is observed at $\sim 1390$ and $1630 \mathrm{~cm}^{-1}$ in the standard solutions. These two regions appear in the Raman spectra for both release and potency sets. Both the $\sim 1390$ and $1630 \mathrm{~cm}^{-1}$ regions show strong correlation with changing ciprofloxacin concentration and follow the trend perfectly.

The calibration standard solutions were used to develop a Raman calibration model for the HPLC experiments. The linear fit based on the measured concentrations and the associated figures of merit for the model are listed in Table 1. The model produces an $\mathrm{R}^{2}$ value for the data of 0.998 . The batch samples were prepared to have concentrations of $\sim 0.2 \mathrm{mg} / \mathrm{ml}$, which lies within the linearity range of the model. The Raman calibration model was used to predict the \% LC from the Raman spectrum of each batch. The predicted results (\%LC) for Raman listed in Table 3, were compared to those from the laboratory method[18]. The results for the Raman versus conventional HPLC methods are all within \pm one standard deviation for all five batches. The pooled average, which incorporates the results of all 30 tablets, is calculated to be essentially the same for results obtained by Raman and HPLC but the pooled standard deviation values are greater for the Raman results by a factor of approximately five. One possible reason for this difference between these standard deviation values is due to the nature of the Raman measurement in the disposable cuvette. While the contents of the cuvette and the HPLC vial are taken from the identical solution, Raman spectral collection requires manual placement of the cuvette in the sample holder, which may vary from sample to sample and cause slight differences in the predicted versus actual values. Conversely, the HPLC measurement is performed by an auto sampler extracting the solutions for each sample in a highly reproducible fashion. Despite the greater spread in the Raman results, these findings support the use of portable Raman spectrometers as an analytical tool to determine potency with HPLC-quality accuracy in less than a minute compared to the 20 minute run time per sample injection. The 30 HPLC samples alone were completed in less than 30 minutes by Raman and would have required 10 hours of run time by HPLC. Finally, using a procedure where the 
samples are prepared according to the chromatographic method, immediate confirmatory analysis by HPLC would be possible for any samples failing the initial Raman experiment and enhance overall speed and efficiency of the laboratory.

\section{Conclusion}

This study indicates the potential for implementation of portable Raman-based approaches into the suite of analytical techniques for pharmaceutical analysis. The results obtained using portable Raman coupled with chemometric-based approaches demonstrate the capability of portable Raman spectrometers to support determination of release and potency of finished drug products. The successful use of portable Raman in this case study of ciprofloxacin tablets indicates that there are multiple potential avenues that are worth pursing in future efforts in the areas of process analytical technology[20-23] and surveillance[9]. Combining portable Raman spectroscopy with dissolution methods opens up opportunities to monitor processes without further sample preparation or aliquot removal. This may have application in the area of monitoring real-time API release[24] using small-footprint spectrometers[25] that can be implemented in the continuous process environment[26].

The promising results for the potency determination of ciprofloxacin tablets may assist in enhancing surveillance since samples can be screened rapidly without each tablet undergoing analysis by HPLC. For large amount of samples requiring screening, the method described in this paper could be employed. Samples prepared identically to the HPLC method requirements would undergo analysis by a Raman spectrometer and only samples failing the Raman method would be subject to chromatographic confirmatory analysis. This workflow would increase the amount of samples undergoing testing and free up resources for those requiring most attention. Fielddeployable methods are a subject of future work and require use of solvents and sample preparation procedures that are field deployable - such as aqueous assays that do not involve use of solvents and minimize generation of hazardous waste in the field.

\section{Disclaimer}

This article reflects the views of the authors and should not be construed to represent FDA's views or policies.

\section{Acknowledgments}

This project was supported, in part, by an appointment (C.V.N, C.T., R.T., L.S.L) to the Research Participation Program at the Center for Drug Evaluation and Research administered by the Oak Ridge Institute for Science and Education through an interagency agreement between the U.S. Department of Energy and the U.S. Food 
and Drug Administration. Support for this project was received through the CDER Medical Counter-Measures initiative (MCMi).

Table 1: Calibration Model Parameters for Portable Raman Analysis

\begin{tabular}{|c|c|c|}
\hline & Release (Raman) & Potency (Raman) \\
\hline Technique & Partial Least Squares & Partial Least Squares \\
\hline Spectral Range, $\mathrm{cm}^{-1}$ & $1100-1800$ & $1100-1800$ \\
\hline \# of Calibration Spectra & 18 & 27 \\
\hline $\begin{array}{r}\text { Range of Calibration Samples } \\
(\mathrm{mg} / \mathrm{ml})\end{array}$ & $0.01-1$ & $0.01-1$ \\
\hline Preprocessing & $\begin{array}{l}\text { Smoothing (2nd order, } 31 \text { point } \\
\text { window); SNV }\end{array}$ & $\begin{array}{l}\text { First Derivative (2nd order, } 31 \text { point } \\
\text { window); SNV }\end{array}$ \\
\hline $\mathbf{R}^{2}$ Calibration (Cal.) & 0.999 & 0.999 \\
\hline $\begin{array}{r}\text { Root Mean Squared Error of } \\
\text { Cal. }(\mathrm{mg} / \mathrm{ml})\end{array}$ & 0.006 & 0.009 \\
\hline Cross Validation (CV) & $\begin{array}{l}\text { Venetian Blinds ( } 4 \text { data splits, } 1 \\
\text { sample per split) }\end{array}$ & $\begin{array}{l}\text { Venetian Blinds ( } 5 \text { data splits, } 1 \\
\text { sample per split) }\end{array}$ \\
\hline $\mathbf{R}^{2}$ Cross Validation (CV) & 0.999 & 0.998 \\
\hline $\begin{array}{r}\text { Root Mean Squared Error of } \\
\text { CV }(\mathrm{mg} / \mathrm{ml})\end{array}$ & 0.008 & 0.011 \\
\hline Latent Variables & 3 & 4 \\
\hline
\end{tabular}


Table 2: Percent (\%) drug release rate results obtained using the Portable Raman and UV Methods at each time point based on six tablets. The specification is for NLT $80 \%$ of the labeled amount of ciprofloxacin is dissolved at $30 \mathrm{~min}$.

\begin{tabular}{|c|c|c|c|c|}
\hline \multirow[b]{2}{*}{ Time (min) } & \multicolumn{2}{|c|}{ Batch 1-Raman } & \multicolumn{2}{|c|}{ Batch 1-UV } \\
\hline & Average & Std. Dev & Average & Std. Dev \\
\hline 10 & 100.5 & 2.9 & 107.1 & 4.3 \\
\hline 20 & 103.7 & 2.2 & 108.3 & 3.8 \\
\hline 30 & 104.4 & 2.3 & 108.5 & 4.4 \\
\hline 60 & 105.1 & 2.1 & 112.9 & 8.5 \\
\hline \multirow[b]{2}{*}{ Time (min) } & \multicolumn{2}{|c|}{ Batch 2-Raman } & \multicolumn{2}{|c|}{ Batch 2-UV } \\
\hline & Average & Std. Dev & Average & Std. Dev \\
\hline 10 & 99.8 & 2.3 & 101.7 & 4.2 \\
\hline 20 & 102.4 & 1.0 & 108.3 & 1.1 \\
\hline 30 & 102.6 & 1.5 & 108.2 & 1.1 \\
\hline 60 & 102.6 & 1.8 & 109.3 & 0.9 \\
\hline \multirow[b]{2}{*}{ Time (min) } & \multicolumn{2}{|c|}{ Batch 3-Raman } & \multicolumn{2}{|c|}{ Batch 3-UV } \\
\hline & Average & Std. Dev & Average & Std. Dev \\
\hline 10 & 98.3 & 1.8 & 98.7 & 1.4 \\
\hline 20 & 103.0 & 0.6 & 102.9 & 0.5 \\
\hline 30 & 103.8 & 1.1 & 103.2 & 1.1 \\
\hline 60 & 103.9 & 1.0 & 106.6 & 1.4 \\
\hline \multirow[b]{2}{*}{ Time (min) } & \multicolumn{2}{|c|}{ Batch 4-Raman } & \multicolumn{2}{|c|}{ Batch 4-UV } \\
\hline & Average & Std. Dev & Average & Std. Dev \\
\hline 10 & 97.9 & 3.7 & 111.2 & 13.3 \\
\hline 20 & 103.7 & 1.5 & 104.0 & 7.9 \\
\hline 30 & 104.1 & 1.9 & 107.0 & 8.2 \\
\hline 60 & 105.1 & 1.4 & 114.5 & 8.8 \\
\hline \multirow[b]{2}{*}{ Time (min) } & \multicolumn{2}{|c|}{ Batch 5-Raman } & \multicolumn{2}{|c|}{ Batch 5-UV } \\
\hline & Average & Std. Dev & Average & Std. Dev \\
\hline 10 & 94.2 & 5.4 & 97.8 & 5.4 \\
\hline 20 & 101.2 & 2.0 & 105.1 & 2.2 \\
\hline 30 & 101.7 & 1.4 & 104.9 & 1.8 \\
\hline 60 & 101.7 & 0.7 & 106.3 & 1.0 \\
\hline
\end{tabular}


Table 3: Potency results obtained using the Portable Raman and UV Methods. All values are percent label claim (\% LC). The values for each individual batch are based on six tablets run.

\begin{tabular}{|l|cc|cc|}
\hline & \multicolumn{2}{|c|}{ Raman } & \multicolumn{2}{c|}{ HPLC } \\
& Average & Std. Dev. & Average & Std. Dev. \\
\hline Batch 1 & 99.2 & 3.0 & 99.4 & 1.7 \\
\hline Batch 2 & 97.6 & 4.8 & 98.8 & 0.9 \\
\hline Batch 3 & 95.8 & 3.8 & 98.3 & 0.8 \\
\hline Batch 4 & 102.2 & 6.8 & 99.5 & 1.4 \\
\hline Batch 5 & 101.9 & 4.9 & 100.0 & 0.8 \\
\hline Pooled & $\mathbf{9 9 . 4}$ & $\mathbf{5 . 1}$ & $\mathbf{9 9 . 2}$ & $\mathbf{1 . 2}$ \\
\hline
\end{tabular}




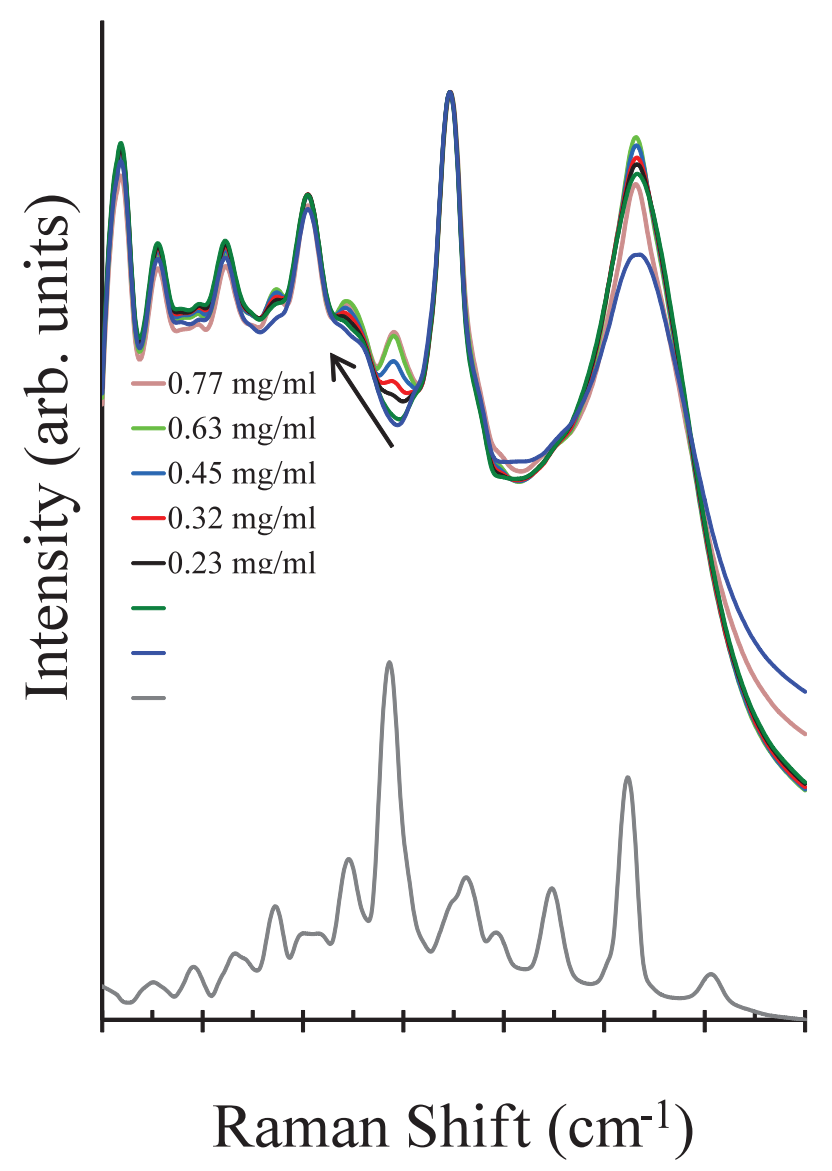

Figure 1. The portable Raman spectra for calibration standard samples used to develop the Raman model for $\%$ drug release. The concentrations are based on the amount of ciprofloxacin present. The arrow indicates the most prominent spectral region where spectral trends are observed for increasing ciprofloxacin content. The Raman spectrum for ciprofloxacin $\mathrm{HCl}$ reference standard is shown for comparison. 

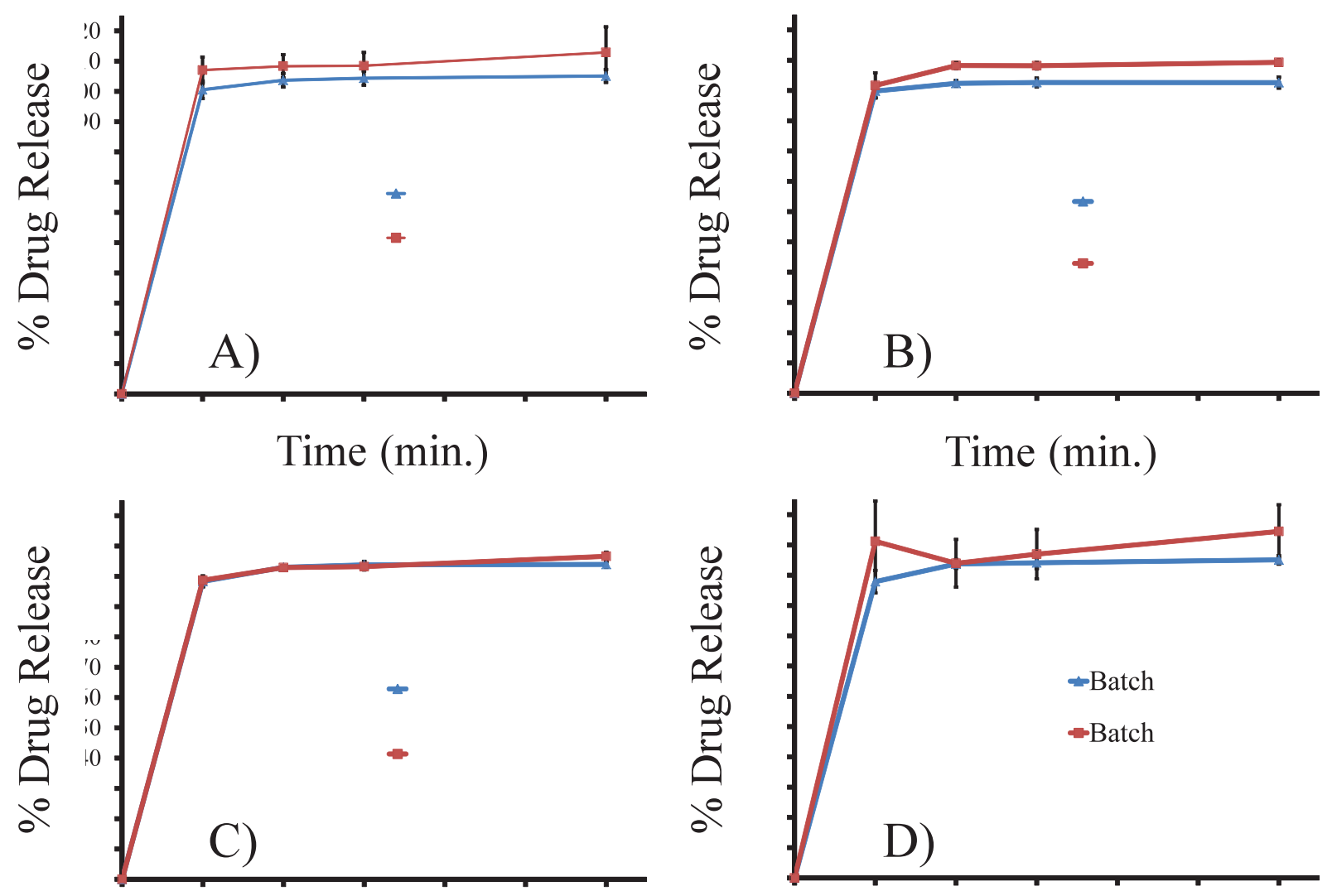

Time (min.)

Time (min.)

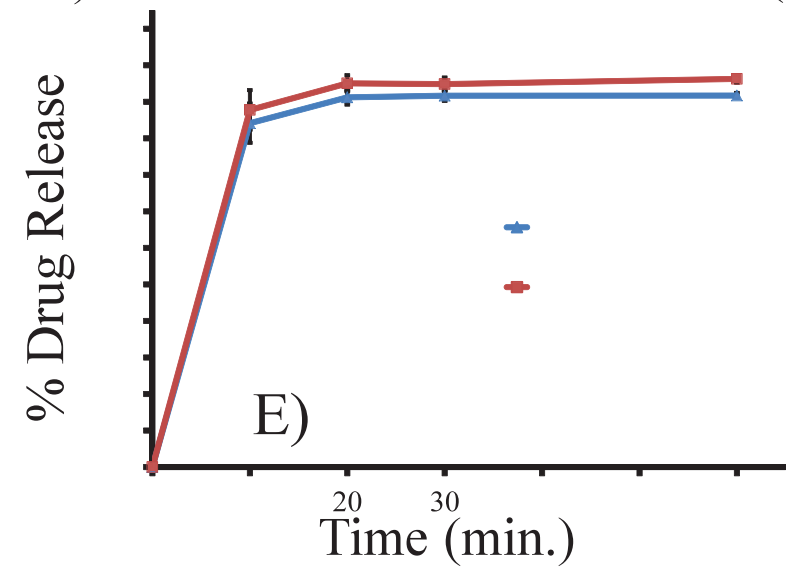

Figure 2. Dissolution Profile comparison for Batches 1-5 (A-E) based on the results of the portable Raman method and UV method. The error bars represent one standard deviation. 

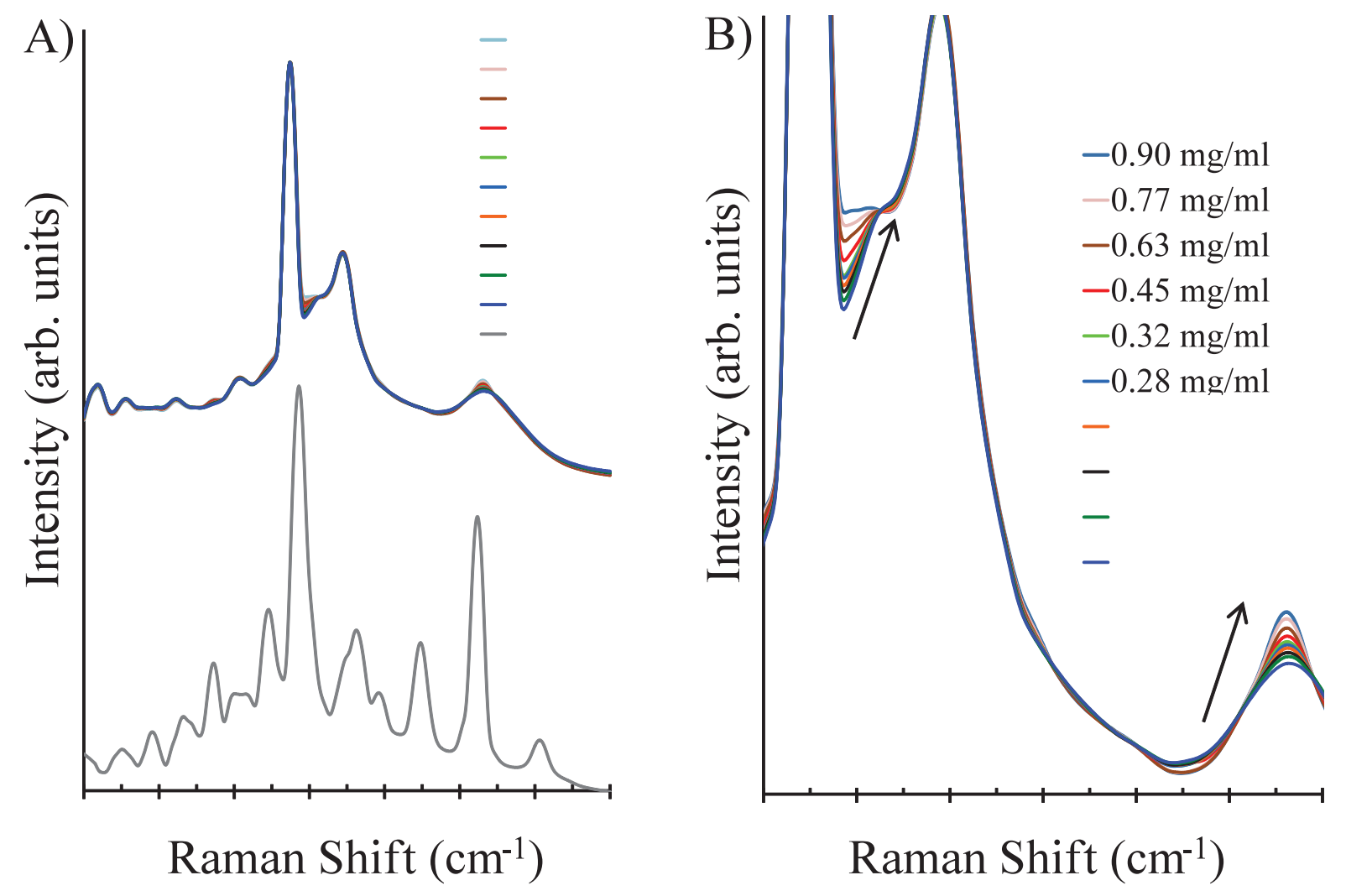

Figure 3. A) The portable Raman spectra for a range of calibration standard samples used to develop the Raman model for potency (\%LC). The concentrations are based on the amount of ciprofloxacin present. The Raman spectrum for ciprofloxacin $\mathrm{HCl}$ reference standard is shown for comparison. B) A closer view of the region between 1350 and $1650 \mathrm{~cm}^{-1}$. The arrows indicate the most prominent spectral regions at $\sim 1400$ and $1625 \mathrm{~cm}^{-1}$ where spectral trends are observed for increasing ciprofloxacin content. 


\section{References}

[1] J.A. DiMasi, Success rates for new drugs entering clinical testing in the United States, Clinical Pharmacology \& Therapeutics 58 (1995) 1-14.

[2] Guideline for submitting samples and analytical data for methods validation., U.S. Food and Drug Administration (1987).

[3] Validation of compendial procedures, U.S. Pharmacopeia 12251 - 5.

[4] J.B. James A. Miller, Crowther, Analytical Chemistry in a GMP Environment, Wiley (May 2000).

[5] J.H.M.M. Joachim Ermer, Method validation in pharmaceutical analysis, Wiley-VCH (2005).

[6] K. Carron, R. Cox, Qualitative analysis and the answer box: a perspective on portable Raman spectroscopy, Anal. Chem. 82 (2010) 3419-3425.

[7] K. Buckley, P. Matousek, Recent advances in the application of transmission Raman spectroscopy to pharmaceutical analysis, Journal of Pharmaceutical and Biomedical Analysis 55 (2011) 645-652.

[8] C.J. Strachan, T. Rades, K.C. Gordon, J. Rantanen, Raman spectroscopy for quantitative analysis of pharmaceutical solids, Journal of Pharmacy and Pharmacology 59 (2007) 179-192.

[9] T. Vankeirsbilck, A. Vercauteren, W. Baeyens, G. Van der Weken, F. Verpoort, G. Vergote, J.P. Remon, Applications of Raman spectroscopy in pharmaceutical analysis, TrAC Trends in Analytical Chemistry 21 (2002) 869-877.

[10] M.J. Pelletier, Quantitative Analysis Using Raman Spectrometry, Applied Spectroscopy 57 (2003) 20A-42A.

[11] M.C. Hennigan, A.G. Ryder, Quantitative polymorph contaminant analysis in tablets using Raman and near infra-red spectroscopies, Journal of Pharmaceutical and Biomedical Analysis 72 (2013) 163-171.

[12] X.-F. Zhang, M.-Q. Zou, X.-H. Qi, F. Liu, C. Zhang, F. Yin, Quantitative detection of adulterated olive oil by Raman spectroscopy and chemometrics, J. Raman Spectrosc. 42 (2011) 1784-1788.

[13] C.M. Gryniewicz-Ruzicka, S. Arzhantsev, L.N. Pelster, B.J. Westenberger, L.F. Buhse, J.F. Kauffman, Multivariate Calibration and Instrument Standardization for the Rapid Detection of Diethylene Glycol in Glycerin by Raman Spectroscopy, Applied Spectroscopy 65 (2011) 334341.

[14] A. Paudel, D. Raijada, J. Rantanen, Raman spectroscopy in pharmaceutical product design, Advanced Drug Delivery Reviews 89 (2015) 3-20.

[15] R. Davis, A. Markham, J.A. Balfour, Ciprofloxacin. An updated review of its pharmacology, therapeutic efficacy and tolerability, Drugs 51 (1996) 1019-1074.

[16] B. Courtney, J. Easton, T.V. Inglesby, C. SooHoo, Maximizing State and Local Medical Countermeasure Stockpile Investments through the Shelf-Life Extension Program, Biosecurity and Bioterrorism: Biodefense Strategy, Practice, and Science 7 (2009) 101-107.

[17] $\mathrm{H}$. Aronson, Correction factor for dissolution profile calculations, Journal of Pharmaceutical Sciences 82 (1993) 1190-1190.

[18] Pharmacopeial Forum, U.S. Pharmacopeia 35843.

[19] S. Matero, F.v. Den Berg, S. Poutiainen, J. Rantanen, J. Pajander, Towards better process understanding: Chemometrics and multivariate measurements in manufacturing of solid dosage forms, Journal of Pharmaceutical Sciences 102 (2013) 1385-1403.

[20] Guidance for industry, PAT - a framework for innovative pharmaceutical development manufacturing, and quality assurance, U.S. Food and Drug Administration (September 2004).

[21] K.A. Esmonde-White, M. Cuellar, C. Uerpmann, B. Lenain, I.R. Lewis, Raman spectroscopy as a process analytical technology for pharmaceutical manufacturing and bioprocessing, Analytical and Bioanalytical Chemistry (2016) 1-13. 
[22] S.L. Lee, T.F. O'Connor, X. Yang, C.N. Cruz, S. Chatterjee, R.D. Madurawe, C.M.V. Moore, L.X. Yu, J. Woodcock, Modernizing Pharmaceutical Manufacturing: from Batch to Continuous Production, Journal of Pharmaceutical Innovation 10 (2015) 191-199.

[23] J. Rantanen, Process analytical applications of Raman spectroscopy, Journal of Pharmacy and Pharmacology 59 (2007) 171-177.

[24] B. Wan, C.A. Zordan, X. Lu, G. McGeorge, In-line ATR-UV and Raman Spectroscopy for Monitoring API Dissolution Process During Liquid-Filled Soft-Gelatin Capsule Manufacturing, AAPS PharmSciTech 17 (2016) 1173-1181.

[25] G. Allison, Y.T. Cain, C. Cooney, T. Garcia, T.G. Bizjak, O. Holte, N. Jagota, B. Komas, E. Korakianiti, D. Kourti, R. Madurawe, E. Morefield, F. Montgomery, M. Nasr, W. Randolph, J.-L. Robert, D. Rudd, D. Zezza, Regulatory and Quality Considerations for Continuous Manufacturing May 20-21, 2014 Continuous Manufacturing Symposium, Journal of Pharmaceutical Sciences 104 (2015) 803-812.

[26] Jestel, N.L. "Raman Spectroscopy" in Process Analytical Technology: Spectroscopic Tools and Implementation Strategies for the Chemical and Pharmaceutical Industries, $2^{\text {nd }}$ edition, Sussex, UK; Wiley 2010, K.A. Bakeev, ed. 
Portable Raman
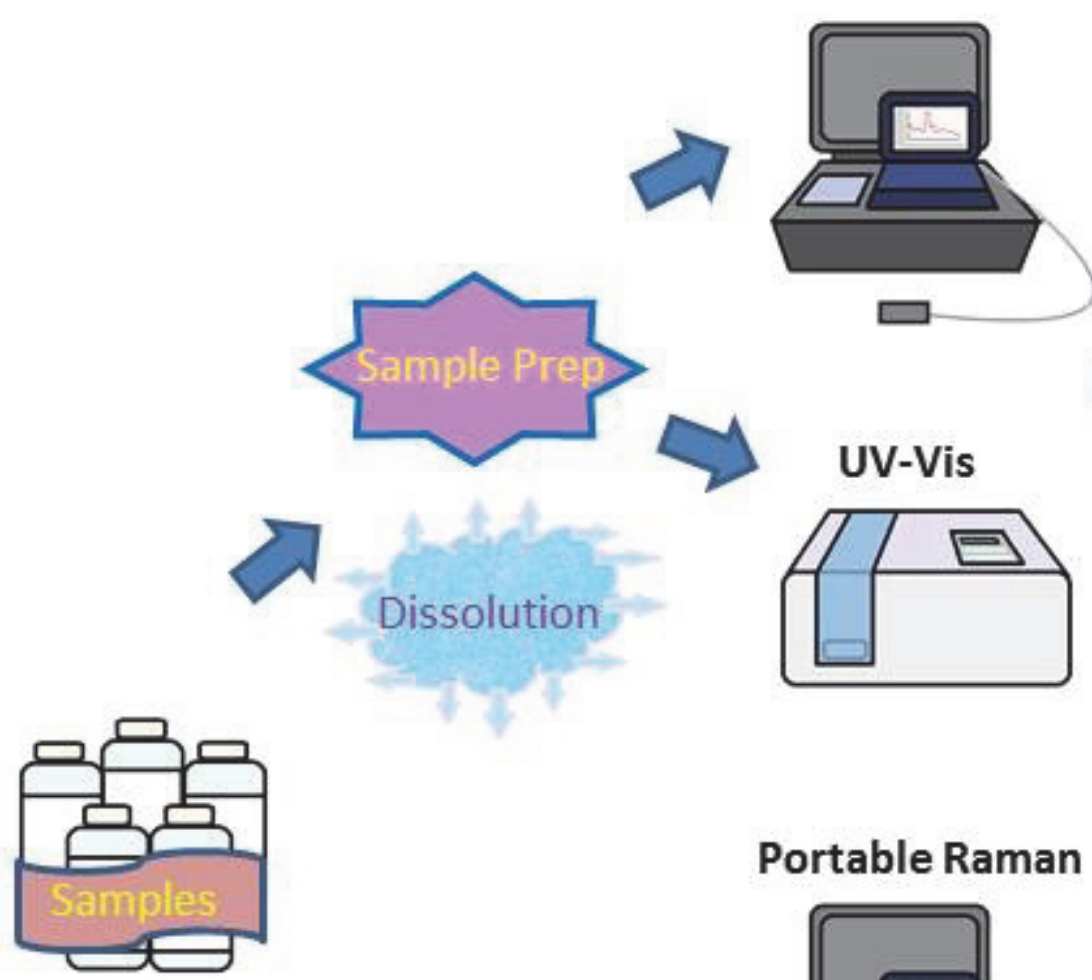

$\triangle$
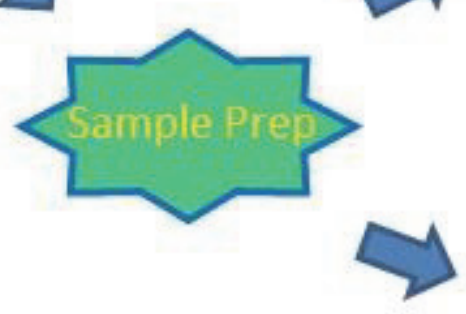

\section{Portable Raman}

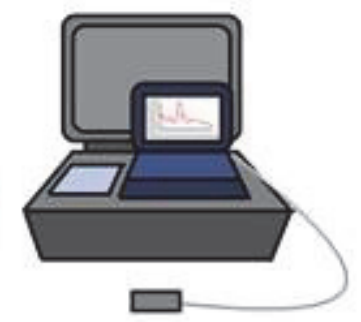

HPLC

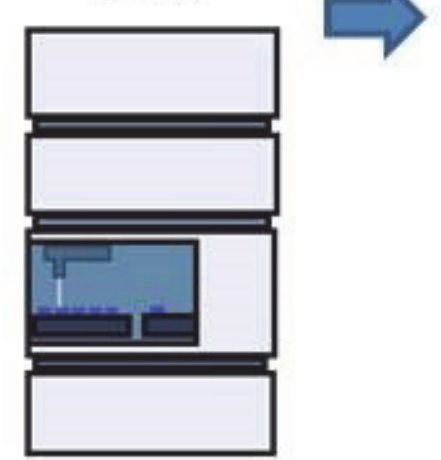

Dissolution Profile Comparison for Batch 5 Based Raman and UV Methods

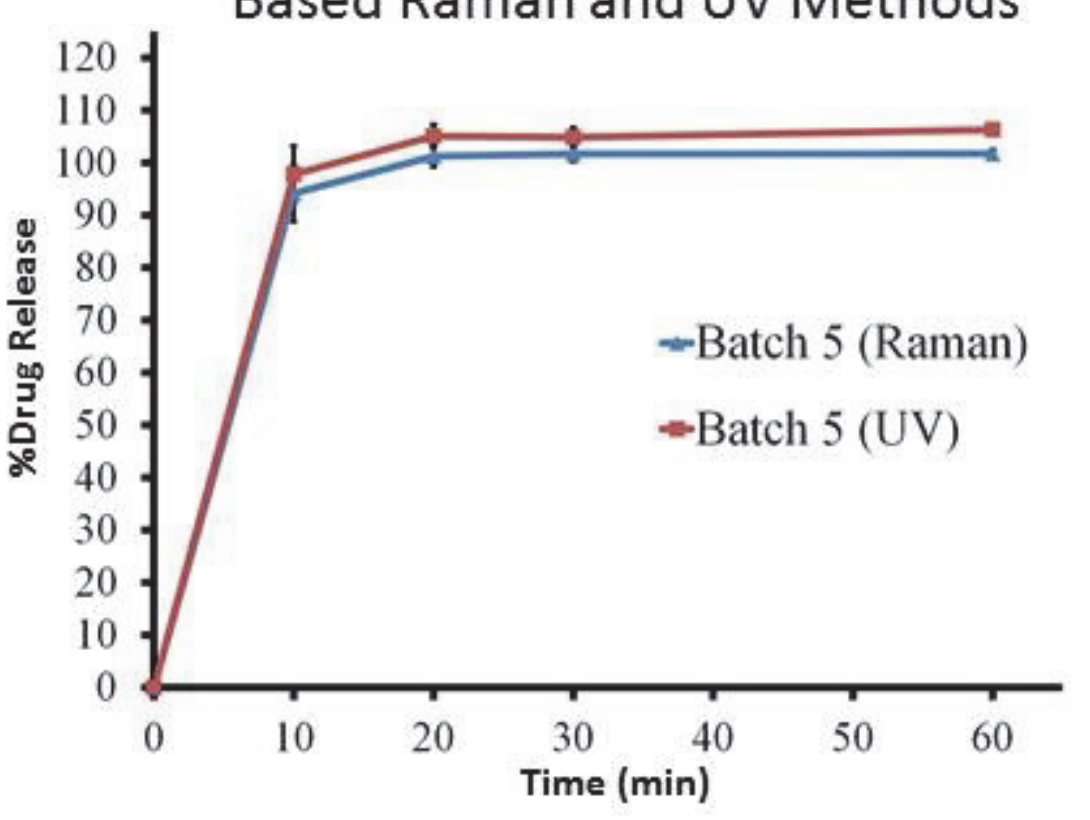

\% Label Claim According to Raman and HPLC Methods

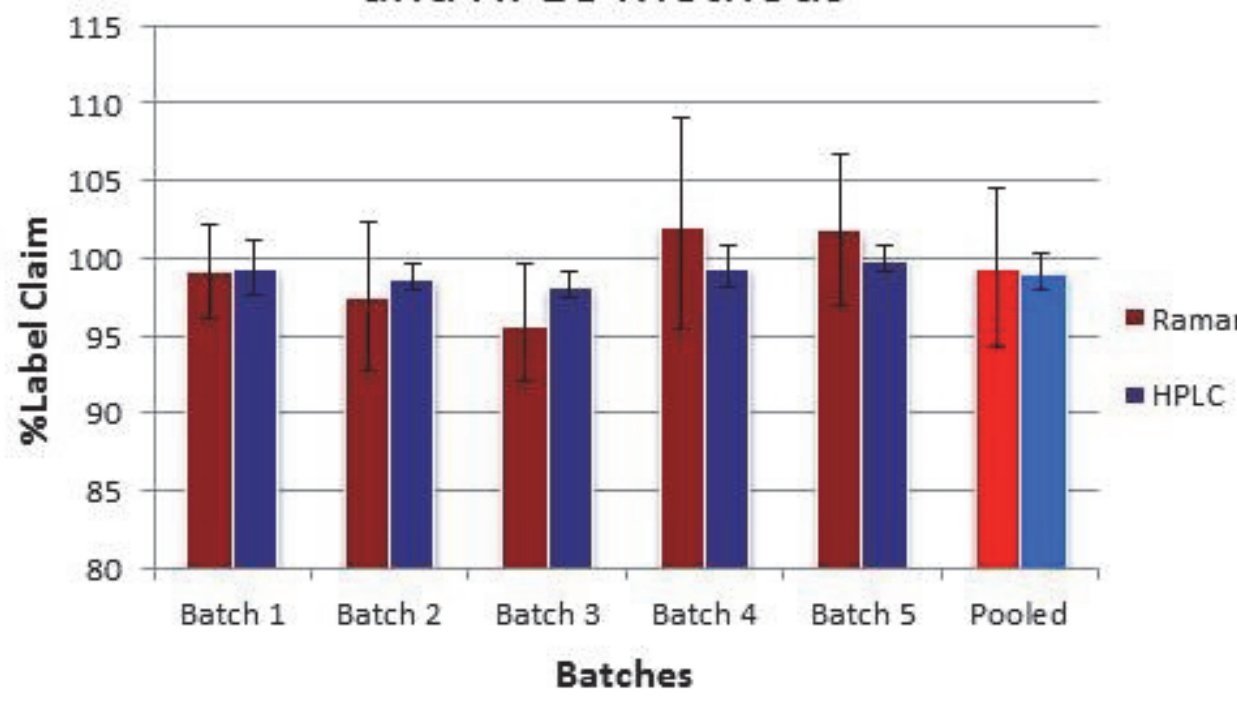

